\title{
Conciencia fonológica en la lectura inicial: Una revisión sistemática
}

\section{Phonological awareness in early reading: A systematic review}

http://dx.doi.org/10.17981/cultedusoc.13.1.2022.04

Recibido: 26 de febrero de 2021. Aceptado: 8 de junio de 2021. Publicado: 24 de enero de 2022 .

\author{
Roxana Angela Flores-Flores (i) \\ Universidad César Vallejo. Lima (Perú) \\ roxana82peru@gmail.com
Yolanda Josefina Huayta-Franco
Universidad César Vallejo. Lima (Perú) yolandahuaytafranco2014@gmail.com
Ana Isabel Galindo-Quispe (iD
Universidad César Vallejo. Lima (Perú) galindoana016@gmail.com
Carolina del Pilar López-Ruiz (iD
Universidad César Vallejo. Lima (Perú)
cadelpizz@gmail.com
Janeth Rosario Gutiérrez-Rojas
Universidad César Vallejo. Lima (Perú) acuarius262@hotmail.com

Para citar este artículo:

Flores-Flores, R., Huayta-Franco, Y., Galindo-Quispe, A., López-Ruiz, C. y Gutiérrez-Rojas, J. (2021). Conciencia fonológica en la lectura inicial: Una revisión sistemática. Cultura, Educación y Sociedad, 13(1), 61-74. DOI: http://dx.doi.org/10.17981/ cultedusoc.13.1.2022.04

\section{Resumen}

Introducción: Actualmente los colegios privados en el Perú promueven que los niños(as) de 5 años aprendan a leer y escribir e ingresen al nivel primario con dichas capacidades desarrolladas. Sin embargo, los niños(as) todavía no han logrado incrementar el desarrollo de la conciencia fonológica. El presente artículo tiene como propósito analizar los aportes de la literatura científica para la comprensión del tema: desarrollo de la conciencia fonológica en la lectura de los niños de 3 a 5 años cursantes de educación inicial en países americanos y europeos. Metodología: Se trabaja una revisión sistemática a través del método de la Declaración PRISMA, en la búsqueda de artículos científicos desde cuatro motores on line: Scopus, ProQuest, EBSCO y Concytec, usando los descriptores: "conciencia fonológica", "educación-preescolar" a su vez combinando entre ellos el uso del booleano AND, en español e inglés. El estudio considera como categorías de análisis: la conciencia fonológica, rendimiento lector, decodificación lectora y estrategias didácticas. Resultados y discusión: Los resultados evidencian el análisis de 12 artículos científicos, de los cuales 4 fueron de Scopus, 3 de ProQuest, 3 de EBSCO, 2 de Concytec y al mismo tiempo 7 fueron artículos con diseños cuasi experimentales, 3 correlacionales, 1 preexperimental y 1 de diseño mixto. Conclusiones: El desarrollo de la conciencia fonológica esta ligada al aprendizaje inicial de la lectura, develando dificultades lectoras en el estudiante, al mismo tiempo, se incentiva la conciencia fonológica al tener palabras cuya decodificación permite convertirlas en sonidos.

Palabras clave: Conciencia fonológica; educación inicial; lenguaje hablado; lingüística; revisión sistemática

\section{Abstract}

Introduction: Currently, private schools in Peru promote that 5-year-old children learn to read and write and enter the primary level with these developed capacities. However, children have not yet managed to increase the development of phonological awareness. The purpose of this article is to analyze the contributions of the scientific literature for the understanding of the subject: development of phonological awareness in the reading of children from 3 to 5 years old in early childhood education in American and European countries. Methodology: A systematic review is carried out through the method of the PRISMA Declaration, in the search for scientific articles from four online engines: Scopus, ProQuest, EBSCO and Concytec, using the descriptors: "phonological awareness", "preschooleducation" at your option. sometimes combining the use of the boolean AND, in Spanish and English. The study considers as categories of analysis: phonological awareness, reading performance, reading decoding and teaching strategies. Results and discussion: The results show the analysis of 12 scientific articles, of which 4 were from Scopus, 3 from ProQuest, 3 from EBSCO, 2 from Concytec and at the same time 7 were articles with quasi-experimental designs, 3 correlational, 1 preexperimental and 1 design mixed. Conclusions: The development of phonological awareness is linked to the initial learning of reading, revealing reading difficulties in the student, at the same time, phonological awareness is encouraged by having words whose decoding allows them to be converted into sounds. Keywords: Learning; phonological awareness; early education; reading; systematic review 


\section{INTRODUCCIÓN}

Actualmente muchas de las investigaciones sobre la enseñanza aprendizaje de la lecto escritura han centrado su fundamentación en los procesos lingüísticos. Si bien es cierto el desarrollo de la escritura va ir acompañado con el desarrollo del lenguaje oral; en tal sentido, se incentiva a que cada niño adquiera los sonidos del lenguaje (fonemas), para su desarrollo natural psicológico, el incremento de sus relaciones interpersonales y finalmente fortalecer el rendimiento académico; teniendo en cuenta que el individuo es el único ser vivo que puede organizar ideas, desarrollar procesos de comprensión y emociones mediante el habla.

En lo referido al rendimiento académico, las pruebas internacionales, según la Organización para la Cooperación y el Desarrollo Económico (OCDE, 2010) y el Programa de Evaluación Internacional-PISA se encargan del ranking de los resultados de las pruebas de comprensión lectora y matemáticas en países afiliados a nivel mundial; en tal sentido, en el último informe, la estimación luego del desarrollo de las pruebas expresaron que los 65 estados que desarrollan estas evaluaciones muestran algunas carencias en el área de comunicación, destacan entre los puestos finales países como Panamá, Perú, Argentina, Colombia y Brasil. En este problema se requiere ofrecer herramientas reveladoras en beneficio de los estudiantes (OCDE, 2010).

En el caso concreto de Perú los efectos de la Evaluación Censal de los Estudiantes-ECE para los especialistas del Ministerio de Educación del Perú-MINEDU, revelaron que, de cada diez niños de Educación Básica Regular-EBR del tercer ciclo (segundo grado), siete de ellos no comprenden lo que leen. En el área de comprensión lectora se establecieron cuatro niveles de logro: (1) C en Inicio (cuando el niño muestra un mínimo progreso en la competencia de acuerdo al nivel esperado); (2) B en Proceso (cuando el niño está cercano al nivel esperado hacia la competencia, para lo cual necesita acompañamiento durante un tiempo razonable para lograrlo); (3) A para Logro Esperado (cuando el niño demuestra el nivel esperado hacia la competencia, evidenciando manejo satisfactorio en las tareas propuestas y en el tiempo designado); y (4) AD es Logro Destacado (cuando el niño demuestra un nivel superior a lo esperado hacia la competencia) (MINEDU, 2019).

Actualmente los colegios privados en el Perú promueven que los niños(as) de cinco años aprendan a leer y escribir e ingresen al nivel primario con dichas capacidades desarrolladas. Sin embargo, los niños(as) todavía no han logrado incrementar el desarrollo de la conciencia fonológica, es decir, la habilidad para manejar conscientemente los segmentos sonoros de la lengua (Lema, Tenezaca y Aguirre, 2019). Este desarrollo es considerado, como uno de los factores que mejor predice el aprendizaje de la lectura, dificultades en tareas que requieren conciencia fonológica,-especialmente a nivel de conciencia silábica en niños pre lectores y conciencia fonémica en quienes han recibido instrucción lectora, se relacionan con mayores dificultades o retrasos en el aprendizaje de la lectura (Bravo, Villalón y Orellana, 2006).

Es necesario para el aprendizaje de la lecto escritura que el niño tome conciencia de los fonemas (conocimiento fonémico) las cuales integran las palabras, y de esa manera al hacerse consciente de la comunicación en sus unidades mínimas, mayor es la facilidad de fijar los fonemas (sonidos) a un grafema (escritura), incentivando de esta forma el 
proceso de asociación entre el sonido y su manifestación gráfica. El aprendizaje de la lectura comienza con el lenguaje, más aún, en la edad preescolar que constituye una edad sensitiva; este aprendizaje se manifiesta paulatinamente, pasando por todas las etapas del proceso y al presentarse trabas en el aprendizaje de la lectura, se evidencian, las limitaciones, evidenciamos de desarrollar las destrezas lingüísticas determinadas durante el periodo de formación inicial o etapa pre escolar (Borzone, 2005). En tal sentido, la conciencia fonológica es la destreza para conocer y manipular los sonidos de la lengua, es el principal pionero del rol lector. En cambio, no se evidencia el uso sistemático de planes de participación pedagógica, por parte de los docentes en la conciencia fonológica, dentro de los salones de niños en etapa pre escolar dirigido a acrecentar tal destreza.

Los conocimientos fonológicos que los estudiantes poseen están estrechamente relacionados con el desarrollo del lenguaje oral en las fases iniciales de su desarrollo vital, por tanto, constituye un factor primordial al inicio de su enseñanza, pues accede a fijar las bases para aprendizajes venideros a través de la teoría "gramática universal" (Sellés y Martínez, 2014) plantea que todo individuo tiene de manera innata un Dispositivo de Adquisición del Lenguaje-LAD, el cual le permite adquirir y desarrollar una lengua asimilando estructuras comunicativas y lingüísticas. Es así que los niños llegan al colegio con saberes previos para la iniciación de la lecto escritura, asimismo los aprendizaje que ejecuten procesos están comprometidos con la lectura y escritura, léxica, sintáctica y semántica (Alarcón, León, Soto-Barba y Sáez, 2021), los cuales se pueden repotenciar mediante actividades y vivencias significativas para los niños.

$\mathrm{Al}$ respecto, desde una perspectiva socio constructivista, el lenguaje es un proceso cognitivo cuyo óptimo desarrollo depende de la interacción sociocultural que los estudiantes realicen, la cual, a su vez, les permiten elaboran los procesos de pensamiento necesarios para poder comunicarse de manera oral y escrita. El enfoque de estas teorías permiten comprender que siendo el lenguaje oral un proceso el cual se va perfeccionando a través de las diferentes experiencias de aprendizaje, y para permitir a los estudiantes desarrollarlo en el aula de clases, es imprescindible planificar actividades destinadas a promover la adquisición y dominio de determinadas habilidades (Bravo et al., 2006). Una de ellas constituye la conciencia fonológica, definida como una habilidad metalingüística que permite reconocer los sonidos (fonemas) que componen el lenguaje oral (Owens, 2003).

Cabe mencionar que existen diferentes expresiones las cuales contribuyen a su comprensión, debido a que, el conocimiento fonológico se relaciona directamente con el aprendizaje de la lecto escritura (Ramos y Cuadrado, 2006), necesariamente un adecuado concepto de conocimiento fonológico, es que tiene como objeto pensar y reflexionar sobre su propia lengua, reconociendo que se refiere a un conocimiento metalingüístico.

Cuando el lenguaje es un conocimiento fonológico, dentro de la didáctica o el campo psicológico, emplean terminologías como conciencia fonémica, sensibilidad fonológica, conocimiento segmental o conciencia fonológica (Araya, 2019), y se hace referencia a la problemática terminológica que se presenta en relación al conocimiento fonológico, señalando que existe una sinonimia con la expresión de conciencia fonológica, ya que ambas describen la habilidad de manipular explícitamente las unidades lingüísticas de la palabra hablada. 
Desde una perspectiva constructivista, la conciencia fonológica puede ser definida como Zona de Desarrollo Próximo-ZDP, término a través del cual Vygotski explica la distancia que existe entre el nivel de desarrollo real y potencial de los niños, bajo la mediación de un adulto. De esta manera, en el umbral mínimo del desarrollo de la conciencia fonológica se encuentran las tareas que los estudiantes pueden resolver de manera autónoma, reconocer oralmente las diversas palabras, identificar aquellas que tienen terminaciones que riman de las que no, y en el umbral máximo, las tareas más dificultosas como establecer semejanzas de palabras que riman en el lenguaje escrito, para lo cual se requiere haber desarrollado habilidades de identificación y segmentación de los fonemas; estos retos puede que no les resulten del todo satisfactorios, aunque cuenten con ayuda mediada.

Es prioridad determinar el umbral mínimo, con la cual puede iniciar la instrucción, por lo que incide la obligación de realizar una evaluación de diagnóstico del nivel del proceso fonológico logrado anteriormente por los niños, antes de establecer los puntos de transición, los cuales se relacionan con el propósito del aprendizaje deseado. Esto determinaría un "umbral" fonológico para el aprendizaje inicial de la lectura (Bravo et al., 2006). En la misma línea, la conciencia fonológica es conceptualizada como la competencia de tomar conciencia y manipular las cualidades estructurales más destacadas del lenguaje en el nivel fonológico.

Entre estas unidades del lenguaje se especifican las sílabas, las unidades intrasilábicas y los fonemas; en tal sentido, las destrezas metalingüísticas implican meditar y manipular los segmentos estructurales de la lengua hablada (Gutiérrez-Fresneda, De Vicente-Yagüe, y Alarcón, 2020). Asimismo, la conciencia fonológica es fracción de la consciencia metalingüística o capacidad para meditar, examinar y manipular los elementos formales del lenguaje en correlación a su fonología. La conciencia fonológica abarca variados roles de dificultad, desde un rol de reconocimiento de sonidos variados hasta un rol más complicado, que concede omitir o agregar características propias, de una educación formal.

La conciencia fonológica aparece de manera gradual durante los primeros años de educación pre escolar (Bravo et al., 2006), es decir, entre los tres y cinco años, la mayoría de los niños tendrían aspectos elementales en esta área durante sus estudios en kindergarten o jardín de niños. En tal sentido, se puede afirmar que la conciencia fonológica, atribuye al niño tomar conciencia de los componentes del lenguaje oral (fonemas, sílabas, palabras) y manipularlos voluntariamente en las diferentes situaciones de lectura y escritura y cuyo desarrollo está estrechamente relacionado con el desarrollo de su lenguaje oral.

El propósito de este artículo es analizar los aportes de la literatura científica para la comprensión del tema: desarrollo de la conciencia fonológica en la lectura de los niños de tres a cinco años, cursantes de educación inicial en países americanos y europeos entre los años 2010 al 2020. Resulta relevante el estudio, debido a la necesidad de aportar conocimiento que conlleve a ofrecer respuestas a todos aquellos niños que ingresan por primera vez al sistema educativo, en el nivel inicial, para luego acceder al primer grado del nivel primaria, con riesgos de mostrar complicaciones en el aprendizaje de las primeras palabras escritas. 


\section{Metodología}

La investigación se circunscribe en un paradigma positivista, enfoque cualitativo, diseño revisión sistemática (Fernández, Baptista y Hernández, 2018). Se parte de una exploración de la literatura científica, respondiendo a la pregunta ¿Cuáles son los aportes científicos para el desarrollo de la conciencia fonológica en la lectura inicial de los niños de tres a cinco años?, usando como método la guía PRISMA (Preferred Reporting Items for Systematic Reviews and Meta Analyses) (Page et al., 2021). Además de ello, se tuvo en cuenta aspectos, como la identificación, selección y análisis de las diversas investigaciones publicadas en las revistas indizadas (Contreras, Páramo y Rojano, 2020). La búsqueda electrónica se realizó en base de datos on line Scopus, ProQuest, EBSCO y Concytec, por ser de acceso institucional, de alta confiabilidad en la calidad académica de sus artículos publicados y poseer material multidisciplinario; esta búsqueda se realizó entre el 1 de setiembre al 19 de diciembre del 2020. Se emplean los siguientes descriptores de búsquedas tanto en español: "conciencia fonológica", "educación pre-escolar", como en inglés: "phonological awareness", "preschool education", a su vez combinando entre ellos el uso del booleano AND.

Los criterios de exclusión, fueron por duplicidad, además de no ser artículos científicos y por no corresponder a la temática de interés. Para los criterios de inclusión se revisó el título, resumen, metodología. Así mismo se verifico que los participantes de las investigaciones estén referidos a los infantes de educación inicial de los países americanos y europeos; se toman en consideración resultados de los artículos recuperados en todas las estrategias de búsqueda, además de artículos revisados exhaustivamente por pares ciegos, a texto completo y acceso abierto, desde el año 2010 al 2020; y también se abordan artículos con potencial para responder al objetivo. Luego se realizó el flujo de la revisión sistemática para el proceso de selección de los artículos científicos basado en la guía PRISMA (Urrútia y Bonfill, 2010). Así mismo, se utilizó el gestor Mendeley (Gallegos, Peralta y Guerrero, 2017) para tener mayor rigurosidad, en la selección de la información.

Para el desarrollo de la metodología de la guía PRISMA, en primer lugar, se configuró la fase de identificación de la búsqueda de artículos en inglés, con el descriptor: "phonological awareness", siendo los resultados: Scopus: 3917; ProQuest: 20; ESBCO: 108542 y Concytec: 56347. Luego se realizó la ecuación y el uso del booleano: "phonological awareness" AND "preschool education", siendo los resultados: Scopus: 17; ProQuest: 20; ESBCO: 3584 y Concytec: 1750

En la misma línea, también se realizó la fase de identificación de los artículos en español, con el descriptor: "conciencia fonológica”, siendo los resultados: Scopus: 40; ProQuest: 20; ESBCO: 3645 y Concytec: 1526. Luego se realizó, la ecuación y el uso del booleano: "conciencia fonológica", AND "educación pre-escolar" siendo los resultados: Scopus: 0; ProQuest: 19; ESBCO: 107 y Concytec: 5. Continuando con la guía PRISMA, se procedió con la segunda fase de cribado, considerando en cuenta el título y a la vez la exclusión de los artículos duplicados, siendo los resultados: Scopus: 84; ProQuest: 70; ESBCO: 72 y Concytec: 8; para un total de 234 artículos. En secuencia, se procedió con la tercera fase de idoneidad o elegibilidad, tomando aspectos como el resumen, método, participantes y resultados, siendo los hallazgos: Scopus: 16; ProQuest: 13; ESBCO: 18 y Concytec: 4, para 
un total de 51 artículos. Finalmente se trabajó la cuarta fase de inclusión al referir los hallazgos: Scopus: 4; ProQuest: 3; ESBCO: 3 y Concytec: 2; para un total de 12 artículos para realizar el análisis.

\section{Resultados}

Los resultados de la investigación evidencian los aportes científicos más resaltantes acerca de la conciencia fonológica en la lectura inicial del estudiantado de formación pre-escolar, en las revistas de alto impacto. El flujo demuestra de manera detallada los artículos científicos identificados con sus respectivos porcentajes (Figura 1).

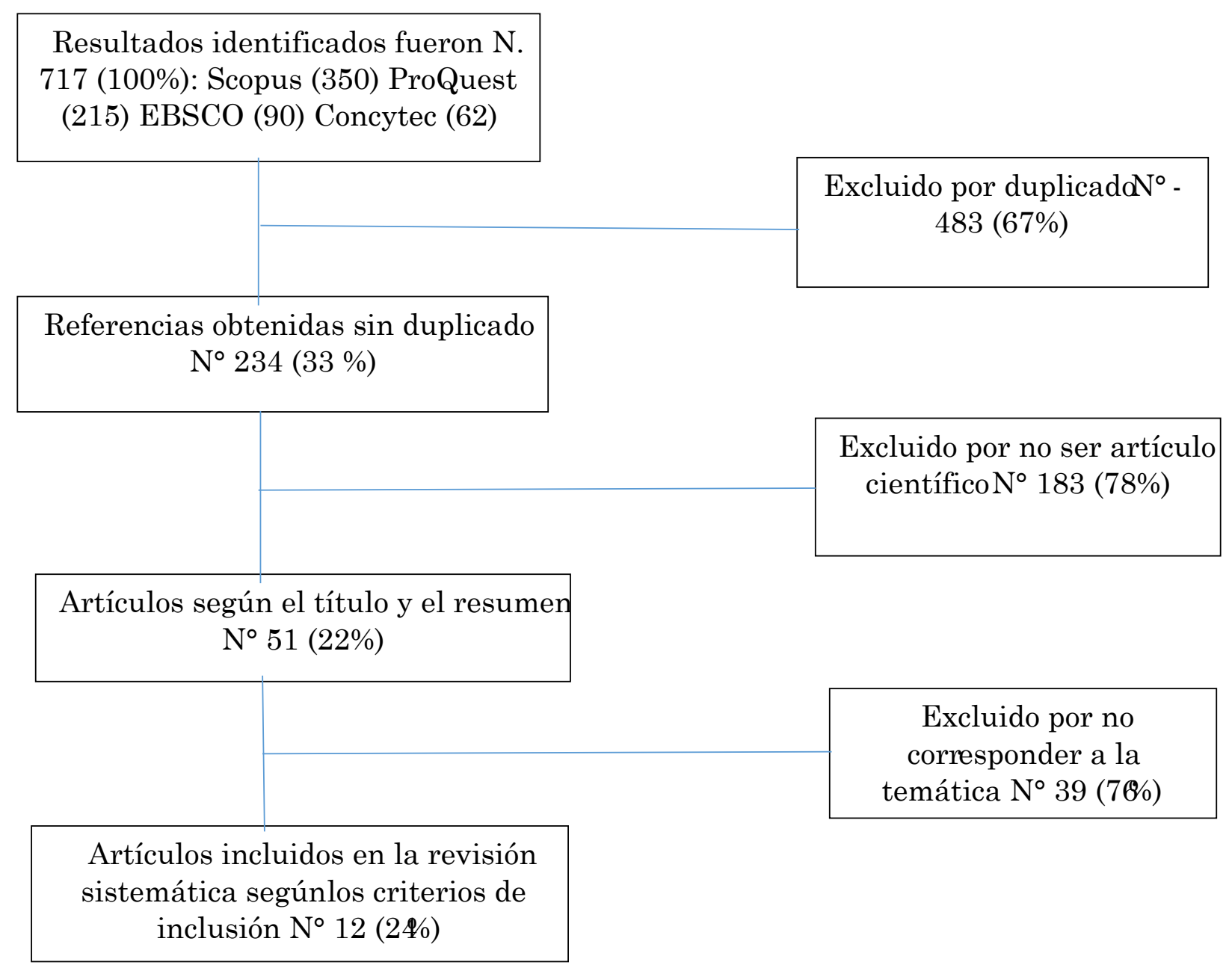

Figura 1. Diagrama de flujo de la revisión sistemática. Fuente: Adaptado de PRISMA.

En el mismo sentido, se presenta la Tabla 1, en la cual, se realizó el análisis de 12 artículos científicos, representando el $24 \%$ del total, proporción muy significativa, por cuanto 4 fueron de Scopus, 3 de ProQuest, 3 de EBSCO, 2 de Concytec y al mismo tiempo 7 fueron artículos con diseños cuasi experimentales, 3 no experimentales correlacionales, 1 pre experimental y 1 de diseño mixto, evidenciándose que todos los estudios pertenecen al enfoque cuantitativo de la investigación. 
TABLA 1.

\section{Artículos analizados}

\begin{tabular}{|c|c|c|c|c|c|c|}
\hline $\mathrm{N}^{\circ}$ & Titulo & Autor/Año & País & Muestra & $\begin{array}{c}\text { Diseño } \\
\text { Metodología }\end{array}$ & Indización \\
\hline 1 & $\begin{array}{l}\text { Nivel de conciencia } \\
\text { fonológica en estudiantes } \\
\text { con funcionamiento } \\
\text { intelectual limítrofe. }\end{array}$ & $\begin{array}{l}\text { (Muñoz-Oyarce } \\
\text { et al., 2020) }\end{array}$ & Chile & $\begin{array}{l}\text { Participantes } 37 \\
\text { estudiantes de la } \\
\text { inicial. }\end{array}$ & $\begin{array}{l}\text { No } \\
\text { experimental, } \\
\text { correlacional }\end{array}$ & Scopus \\
\hline 2 & $\begin{array}{l}\text { Temporo-frontal } \\
\text { activation during } \\
\text { phonological processing } \\
\text { predicts gains in } \\
\text { arithmetic facts in young } \\
\text { children. }\end{array}$ & $\begin{array}{l}\text { (Suárez- } \\
\text { Pellicioni et al., } \\
\text { 2019) }\end{array}$ & EE. UU. & 42 de 110 niños. & $\begin{array}{l}\text { Cuasi } \\
\text { experimental }\end{array}$ & Scopus \\
\hline 3 & $\begin{array}{l}\text { Efecto de un programa } \\
\text { de estimulación de la } \\
\text { conciencia fonológica } \\
\text { en niños preescolares: } \\
\text { sensibilidad a la rima y } \\
\text { a la segmentación. }\end{array}$ & $\begin{array}{l}\text { (Suárez-Yepes et } \\
\text { al., 2019) }\end{array}$ & Colombia & $\begin{array}{l}69 \text { niños entre } 3 \text { y } 4 \\
\text { años de edad. }\end{array}$ & $\begin{array}{l}\text { Cuasi } \\
\text { experimental }\end{array}$ & EBSCO \\
\hline 4 & $\begin{array}{l}\text { Libros en mano: } \\
\text { Phonological awareness } \\
\text { intervention in children's } \\
\text { native languages. }\end{array}$ & $\begin{array}{l}\text { (Gonzales \& } \\
\text { Tejero, 2018) }\end{array}$ & EE. UU. & $\begin{array}{l}\text { Cuatro niños en } \\
\text { edad preescolar ( } 3 \\
\text { niños, } 1 \text { niña). }\end{array}$ & Mixta & Scopus \\
\hline 5 & $\begin{array}{l}\text { Efectos de la } \\
\text { comunicación dialógica y } \\
\text { la conciencia fonológica } \\
\text { en el aprendizaje inicial } \\
\text { de la escritura de } \\
\text { palabras en español. }\end{array}$ & $\begin{array}{l}\text { (Gutiérrez- } \\
\text { Fresneda, 2017) }\end{array}$ & España & $\begin{array}{l}448 \text { alumno de } 5 \text { y } \\
6 \text { años de edad. }\end{array}$ & $\begin{array}{l}\text { Cuasi } \\
\text { experimental }\end{array}$ & EBSCO \\
\hline 6 & $\begin{array}{l}\text { Efectos de la lectura } \\
\text { compartida y la } \\
\text { conciencia fonológica } \\
\text { para una mejora en el } \\
\text { aprendizaje lector. }\end{array}$ & $\begin{array}{l}\text { (Gutiérrez- } \\
\text { Fresnada, 2016) }\end{array}$ & España & $\begin{array}{l}\text { Participaron } 424 \\
\text { alumnos con edades } \\
\text { comprendidas entre } \\
\text { los } 5 \text { y los } 6 \text { años. }\end{array}$ & $\begin{array}{l}\text { Cuasi- } \\
\text { experimenta }\end{array}$ & ProQuest \\
\hline 7 & $\begin{array}{l}\text { Aprendizaje de la } \\
\text { escritura y habilidades } \\
\text { de conciencia fonológica } \\
\text { en las primeras edades. }\end{array}$ & $\begin{array}{l}\text { (Gutiérrez- } \\
\text { Fresnada \& } \\
\text { Mediavilla, 2015) }\end{array}$ & España & $\begin{array}{l}\text { Participación de } \\
166 \text { alumnos con } \\
\text { edades entre los } 4 \text { y } \\
\text { los } 6 \text { años. }\end{array}$ & $\begin{array}{l}\text { Diseño } \\
\text { correlacional- } \\
\text { predictivo }\end{array}$ & Concytec \\
\hline 8 & $\begin{array}{l}\text { Aplicación de } \\
\text { un programa de } \\
\text { estimulación de la } \\
\text { conciencia fonológica } \\
\text { en preescolares de } \\
\text { nivel transición } 2 \text { y } \\
\text { alumnos de primer año } \\
\text { básico pertenecientes } \\
\text { a escuelas vulnerables } \\
\text { de la provincia de } \\
\text { concepción, Chile. }\end{array}$ & $\begin{array}{l}\text { (Arancibia et al., } \\
\text { 2012) }\end{array}$ & Chile & $\begin{array}{l}20 \text { pre escolares } \\
\text { del nivel transición. }\end{array}$ & $\begin{array}{l}\text { Pre } \\
\text {-experimental }\end{array}$ & Scopus \\
\hline 9 & $\begin{array}{l}\text { Velocidad de nombrar y } \\
\text { conciencia fonológica en } \\
\text { el aprendizaje inicial de } \\
\text { la lectura. }\end{array}$ & $\begin{array}{l}\text { (Aguilar et al., } \\
2012 \text { ) }\end{array}$ & España & $\begin{array}{l}\text { Muestra es } 85 \\
\text { niños. }\end{array}$ & Correlacional & ProQuest \\
\hline
\end{tabular}




\begin{tabular}{|c|c|c|c|c|c|c|}
\hline 10 & $\begin{array}{l}\text { Un programa de } \\
\text { intervención pedagógica } \\
\text { en conciencia fonológica, } \\
\text { efectos sobre el } \\
\text { aprendizaje inicial de la } \\
\text { lectura. }\end{array}$ & (Porta, 2012) & Argentina & $\begin{array}{l}\text { La muestra es de } \\
62 \text { niños de nivel } \\
\text { inicial } 5 \text { años de } \\
\text { edad. }\end{array}$ & $\begin{array}{l}\text { Cuasi- } \\
\text { experimental }\end{array}$ & EBSCO \\
\hline 11 & $\begin{array}{l}\text { Niveles de dificultad de } \\
\text { la conciencia fonológica y } \\
\text { aprendizaje lector. }\end{array}$ & $\begin{array}{l}\text { (Aguilar et al., } \\
\text { 2011) }\end{array}$ & España & $\begin{array}{l}299 \text { estudiantes } \\
\text { de edades } \\
\text { comprendidas entre } \\
5,6 \text { y } 7,6 \text { años de } \\
\text { edad. }\end{array}$ & $\begin{array}{l}\text { Cuiasi- } \\
\text { experimental }\end{array}$ & Concytec \\
\hline 12 & $\begin{array}{l}\text { Desarrollo fonológico } \\
\text { en niños de } 3 \text { y } 4 \text { años } \\
\text { según la fonología } \\
\text { natural: Incidencia de la } \\
\text { edad y del género. }\end{array}$ & $\begin{array}{l}\text { (Coloma et al., } \\
2010)\end{array}$ & Suecia & $\begin{array}{l}\text { Participaron } \\
180 \text { niños en dos } \\
\text { grupos, uno de } 3 \\
\text { años y otro de } 4 \\
\text { años. }\end{array}$ & $\begin{array}{l}\text { Cuasi- } \\
\text { experimental }\end{array}$ & ProQuest \\
\hline
\end{tabular}

Fuente: Elaboración propia.

\section{DisCUSIÓN}

El objetivo del artpiculo es analizar los aportes científicos que explican el desarrollo de la conciencia fonológica en la lectura de los niños de 3 a 5 años del nivel educativo inicial, en estudiantes de países americanos y europeos entre los años 2010 al 2020. Siendo el autor Gutiérrez (2016), el más relevante, puesto que sus aportes investigativos se han producido en forma cronológica, ligadas al aprendizaje lector de los niños de formación pre-escolar español.

$\mathrm{Al}$ analizar la conciencia fonológica y su desarrollo en el rendimiento lector, se evidencia que no es un fenómeno aislado, sino al contrario, es un conjunto de destrezas y habilidades con diversos grados de complejidad y prioridad durante el proceso de aprendizaje (Aguilar, Navarro, Menacho, Alcale, Marchena y Ramiro, 2012). Todo esto contribuye al inicio de la pre lectura de grafemas, pero a la vez no se relaciona con la comprensión de la lectura; en otras palabras, el niño puede leer mecánicamente sin necesariamente comprender lo leído. La conciencia fonológica, detecta los fonemas, los domina, lo concientiza y luego los utiliza, a través de la articulación de palabras, por lo que es imprescindible modular la dificultad de tareas en relación a la escolaridad de los niños.

En el mismo sentido, el valor predictivo de la habilidad de la conciencia fonológica, para adquirir la destreza de la lectura inicial, es necesario la intervención pedagógica de una planificación sistemática que refuerce el desarrollo pre lector de los niños (Porta, 2012). Asimismo, los niños al ingresar al sistema educativo, desde el primer peldaño de escolaridad, debe enfocarse en programas educativos de intervención que incentiven la conciencia fonológica, con el principio que cada letra del alfabeto tiene sonidos diferentes y en su conjunto forman nuevas palabras, para que, el nuevo pre lector tenga seguridad en el aprendizaje de la lecto escritura.

Asimismo, el proceso de la habilidad de la conciencia fonológica, se incrementa a través de estrategias o intervenciones, a estudiantes con trabas en el aprendizaje de la lecto escritura (Aguilar, Marchena, Navarro, Menacho y Alcalde, 2011). En tal sentido se utilizó 
la estrategia de la velocidad de nombrar palabras, ya que, apoya de forma diferente a la decodificación de la lectura, al mismo tiempo eleva la calidad del rendimiento académico de los niños, confirmando el mejoramiento de aquellos con ciertas dificultades en su aprendizaje.

En la misma línea, otro programa de intervención pedagógica, basado en el proceso de comunicación dialógica, fortalece la habilidad de la conciencia fonológica en niños de edad pre escolar (Gutiérrez-Fresneda, 2017). Dados los resultados de las trabas del aprendizaje de la escritura, es necesario implementar programas de intervención docente en niños de 5 a 6 años, el cual incentive la conciencia fonológica, a través de objetivos didácticos que favorezcan el proceso léxico y active el mejoramiento de la lecto escritura. Asimismo, el programa de intervención pedagógica fue la estimulación de la sensibilidad de la rima y su segmentación (Suárez-Yepes, Sourdis, Harb y De los Reyes-Aragón, 2019; Arancibia, Bizama y Sáez, 2012). Igualmente este programa mejoró la habilidad de la conciencia fonológica, mediante la aplicación de esta estrategia didáctica, generando condiciones adecuadas para un mejor aprendizaje, beneficiando así su desarrollo a través de una lectura concordante.

En tal sentido, también se considera a la práctica de lectura compartida, como una estrategia para desarrollar la habilidad de la conciencia fonológica, en niños de nivel inicial (Gutiérrez, 2016), y con ello se ha conseguido, la facilitación y el mejoramiento del aprendizaje de la lectura y su comprensión, alcanzando un óptimo desarrollo en el aspecto fonológico y ortográfico del uso. del lenguaje, permitiendo fortalecer el aprendizaje lector desde edades muy tempranas.

También las representaciones fonológicas, juegan un papel fundamental en el aprendizaje del área de matemáticas con la aplicación de la estrategia de juicio de rimas (SuárezPellicioni, Fuchs \& Booth, 2019) en tal sentido, se puede afirmar específicamente que la habilidad fonológica en los niños más pequeños, es importante cultivarlo desde las primeras etapas del desarrollo de las matemáticas, aunque esa habilidad parece poseer efectos que involucran la recuperación de información que se acumulan como códigos fonológicos en la memoria.

Incluso, la habilidad de la conciencia fonológica está presente también en niños con funcionamiento intelectual limítrofe, solo que muestran variadas complicaciones que afectan su destreza académica (Muñoz-Oyarce, Monzalve-Macaya, Almonacid-Fierro y MerellanoNavarro, 2020). Por ésta razón también, se deben impulsar programas especializados tempranos de intervención docente, en los cuales estos infantes logren la habilidad de la conciencia fonológica, mediante herramientas pedagógicas de integración no solo social sino también cultural, para que logren el aprendizaje aunque sea lento, de la lecto escritura y evitar la deserción estudiantil.

Es más, en la conciencia fonología de estudiantes de jardín de niños cuya lengua materna es el inglés, y que desean aprender español, la intervención pedagógica de alfabetización emergente, resulto sorprendentemente efectivo (Gonzales y Tejero, 2018). A través de ello, se evidencia el aumento en las habilidades de conciencia fonológica en inglés y en español, para la segmentación de las sílabas y los sonidos de cada vocal y consonante, y a la vez motivaron un mejoramiento en la lectura. 
Otro aspecto, que se debe tener en cuenta, son los subprocesos de simplificación de la conciencia fonológica que tienden a decrecer en forma significativa, con el paso de los años (Coloma, Pavez, Maggiolo y Peñaloza, 2010; Gutiérrez y Díez, 2015). En otras palabras, estos subprocesos son prioritariamente estructurales y no están relacionados con el género ni la edad de los niños, pero si está relacionado con la adquisición de nuevos fonemas, los cuales sirven para el enriquecimiento del vocabulario de los niños.

El refuerzo de la decodificación lectora en sus inicios, se manifiesta en las habilidades que permiten la obtención de la conciencia fonológica en las primeras fases del proceso educativo (Gutiérrez y Díez, 2015) por lo cual se evidencia una relación muy significativa entre las habilidades de la conciencia fonológica y el aprendizaje de la lecto escritura en niños de edad de 4 a 6 años, sobre las mínimas unidades de los fonemas y la sílaba.

Una limitación en esta investigación, es que en los artículos analizados, no se evidencia el seguimiento a los niños, para verificar si los resultados alcanzados en los diversos artículos científicos, se sustentan en el tiempo, puesto que, la conciencia fonológica constituye el gran predictor para el aprendizaje de la lectura; por lo que se sugiere continuar con investigaciones que permitan validar la sustentabilidad, y poder develar la influencia de la conciencia fonológica en el aprendizaje de la lecto escritura, en las primeras etapas de la escolaridad.

\section{Conclusiones}

Se concluyó que hay evidencias de la conciencia fonológica que tiene relación directa con el aprendizaje inicial de la lectura, descubriendo trabas lectoras en los estudiantes y a la vez incentivando tener conciencia de las palabras para poder descodificarlas, ya que las palabras se convierten en sonidos.

En tal sentido, las capacitaciones y actualizaciones de los docentes del nivel inicial, jardín de niños o pre escolar, referidos a herramientas, estrategias didácticas o intervenciones docentes, constituyen la piedra angular que sostiene el mejoramiento del aprendizaje lecto escritor de los infantes, puesto que esos docentes se encuentran en los cimientos de la formación de los niños, ya que ellos recién están ingresando al sistema educativo.

La conciencia fonológica permite que los niños reconozcan y usen los sonidos del lenguaje, es una habilidad metalingüística, que ofrece elementos fonológicos del lenguaje oral y escrito, seleccionando palabras que tengan rimas y fortalecer de forma consciente los segmentos del lenguaje hablado.

La conciencia fonológica tiene muchos beneficios, como el aumento del bagaje del vocabulario, la alfabetización constante, mejor manejo de los mensajes verbales, como también aprender otros idiomas diferentes a la de su lengua materna mediante transferencias lingüísticas, mientras más pequeño es el niño su conciencia fonológica se activa para aprender con mucha facilidad.

Por lo expresado, las instituciones educativas de formación inicial son fundamentales, pues los profesores pueden detectar una que otra dificultad que presenten los niños en las etapas tempranas de su escolaridad, evidenciando que las habilidades de la conciencia fonológica de los niños son necesarias, pues de allí se desarrolla la adquisición del correcto lenguaje, y esto a la vez le permitirá progresar en la socialización con los demás individuos. 
Finalmente, acerca de la función destacada de la conciencia fonológica son variadas y decisivas, en tal sentido, se afirma que toda programación y ejecución de estrategias didácticas o programas de intervención pedagógica, se brinden de forma continua y sistemática e incluyan acciones educativas significativas, incentivando la solidez cognitiva necesaria, de los niños en edad pre escolar, al iniciar de su vida en la escuela.

\section{REFERENCIAS}

Aguilar, M., Marchena, E., Navarro, J., Menacho, I. y Alcalde, C. (2011). Niveles de dificultad de la conciencia fonológica y aprendizaje lector. Revista de Logopedia, Foniatria y Audiologia, 31(2), 96-105. https://doi.org/10.1016/S0214-4603(11)70177-2

Aguilar, M., Navarro, J., Menacho, I., Alcale, I., Marchena, E. y Ramiro, P. (2012). Velocidad de nombrar y conciencia fonológica en el aprendizaje inicial de la lectura. Psicothema, 22(3), 436-442. Disponible en http://www.psicothema.com/pi?pii=3749

Alarcón, E., León, H., Soto-Barba, J. y Sáez, K. (2021). Ajustes fonético-fonológicos en niños de primer año de educación básica provenientes del sistema educativo público y privado. Incidencia del sexo y el tipo de establecimiento educacional al que asisten. Boletín de Filología, 56(1), 237-262. Disponible en https://boletinfilologia.uchile.cl/ index.php/BDF/article/view/64210

Arancibia, B., Bizama, M. y Sáez, K. (2012). Aplicación de un programa de estimulación de la conciencia fonológica en preescolares de nivel transición 2 y alumnos de primer año básico pertenecientes a escuelas vulnerables de la provincia de concepción, Chile. Revista Signos, 45(80), 236-256. https://doi.org/10.4067/S0718-09342012000300001

Araya, J. (2019). Los Principios de la Conciencia Fonológica en el Desarrollo de la Lectoescritura Inicial. Revista de Lenguas Modernas, (30), 163-181. Disponible en https:// revistas.ucr.ac.cr/index.php/rlm/article/view/38981

Borzone, A. (2005). La Lectura de Cuentos en el Jardín Infantil: Un Medio Para el Desarrollo de Estrategias Cognitivas y Lingüísticas. Psykhe, 14(1), 192-209. http://dx.doi. org/10.4067/S0718-22282005000100015

Bravo, L., Villalón, M. y Orellana, E. (2006). Diferencias en la predictividad de la lectura entre primer año y cuarto año básicos. Psykhe, 15(1), 1-1. https://doi.org/10.4067/ s0718-22282006000100001

Coloma, C. J., Pavez, M. M., Maggiolo, M. y Peñaloza, C. (2010). Desarrollo fonológico en ninos de 3 y 4 antild;os según la fonología natural: Incidencia de la edad y del género. Revista Signos, 42(72), 31-48. https://doi.org/10.4067/S0718-09342010000100002

Contreras, M., Páramo, D. y Rojano, Y. (2020). La teoría fundamentada como metodología de construcción teórica. Pensamiento y Gestión, 47, 283-306. Disponible en https:// rcientificas.uninorte.edu.co/index.php/pensamiento/article/view/12342

Gallegos, M. C., Peralta, C. A. y Guerrero, W. M. (2017). Utilidad de los gestores bibliográficos en la organización de la información para fines investigativos. Formacion Universitaria, 10(5), 77-85. http://dx.doi.org/10.4067/S0718-50062017000500009 
Gonzales, W. y Tejero, M. (2018). Libros en mano: Phonological awareness intervention in children's native languages. Education Sciences, 8(4), 1-12. https://doi.org/10.3390/ educsci8040175

Gutiérrez F., R. (2016). Efectos de la lectura compartida y la conciencia fonológica para una mejora en el aprendizaje lector. Revista Complutense de Educacion, 29(2), 441454. https://doi.org/10.5209/RCED.52790

Gutiérrez, R. y Díez, A. (2015). Aprendizaje de la escritura y habilidades de conciencia fonológica en las primeras edades. Bordón, 67(4), 43-59. https://doi.org/10.13042/Bordon.2015.67405

Gutiérrez-Fresneda, R. (2017). Efectos de la comunicación dialógica y la conciencia fonológica en el aprendizaje inicial de la escritura de palabras en español. Onomazein, 37(4), 170-187. https://doi.org/10.7764/onomazein.37.10

Gutiérrez-Fresneda, R., De Vicente-Yagüe, M. I. y Alarcón, R. (2020). Desarrollo de la conciencia fonológica en el inicio del proceso de aprendizaje de la lectura. Revista Signos, 53(104), 664-681. https://doi.org/10.4067/s0718-09342020000300664

Fernández, C., Baptista, P. y Hernández, R. (2018). Metodología de la investigación. México, D.F.: Mc Graw-Hill Interamericana.

Lema, R. A., Tenezaca, R. E. y Aguirre, S. Y. (2019). El aprestamiento a la lectoescritura en la educación preescolar. Conrado, 15(66), 244-252. Disponible en http://conrado. ucf.edu.cu/index.php/conrado

Muñoz-Oyarce, M. F., Monzalve-Macaya, M., Almonacid-Fierro, A. y Merellano-Navarro, E. (2020). Nivel de conciencia fonológica en estudiantes con funcionamiento intelectual limítrofe. Avances en Psicología Latinoamericana, 38(2), 1-14. https://doi. org/10.12804/revistas.urosario.edu.co/apl/a.7704

OCDE. (2010). Resultados del Informe PISA 2009: Tendencias de aprendizaje. Cambios en el rendimiento de los estudiantes desde 2000. (Volumen V). Madrid: Santillana. Recuperado de https://www.oecd-ilibrary.org/docserver/9789264177543-es.pdf?expire $\mathrm{s}=1642382227 \& \mathrm{id}=\mathrm{id \& accname}=$ guest\&checksum=006E65DA85171D1EA30865513 $3 \mathrm{EDD} 3 \mathrm{FB}$

Owens, R. (2003). Desarrollo del lenguaje. [5 ed.]. Madrid: Pearson.

Page, M. J., McKenzie, J. E., Bossuyt, P. M., Boutron, I., Hoffmann, T. C., Mulrow, C. D., Shamseer, L., Tetzlaff, J. M., Akl, E. A., Brennan, S. E., Chou, R., Glanville, J., Grimshaw, J. M., Hróbjartsson, A., Lalu, M. M., Li, T., Loder, E. W., Mayo-Wilson, E., McDonald, S., McGuinness, L. A., Stewart, L. A., Thomas, J., Tricco, A. C., Welch, V. A., Whiting, P. \& Moher, D. (2021). The PRISMA 2020 statement: An updated guideline for reporting systematic reviews. International Journal of Surgery, 88, 1-9. https:// doi.org/10.1016/j.ijsu.2021.105906

Porta, M. (2012). Un programa de intervención pedagógica en conciencia fonológica. Efectos sobre el aprendizaje inicial de la lectura. Revista de Orientación Educacional, 26 (50), 93-111. Disponible en https://www.upla.cl/bibliotecas/revistas/roe/vol-26-no50segundo-semestre-ano-2012/ 
Ramos, J. L. y Cuadrado, I. (2006). Prueba para la evaluacion de conocimiento fonologico. Madrid: EOS.

República de Perú. MINEDU. (2019). Diseño Curricular Básico Nacional 2019. [Online]. Disponible en http://www.minedu.gob.pe/superiorpedagogica/categoria-producto/publicaciones/dcbn/dcbn-2019/

Sellés, P. y Martínez, T. (2014). Secuencia evolutiva del conocimiento fonológico en niños prelectores. Revista de Logopedia, Foniatría y Audiología, 34(3), 118-128. https://doi. org/10.1016/J.RLFA.2013.09.001

Suárez-Pellicioni, M., Fuchs, L. \& Booth, J. R. (2019). Temporo-frontal activation during phonological processing predicts gains in arithmetic facts in young children. Developmental Cognitive Neuroscience, 40, 1-17. https://doi.org/10.1016/j.dcn.2019.100735

Suárez-Yepes, N., Sourdis, M., Harb, S. L. y De los Reyes-Aragón, C. (2019). Efecto de un programa de estimulación de la conciencia fonológica en niños preescolares: sensibilidad a la rima y a la segmentación. Psicogente, 22(42), 236-254. https://doi.org/10.17081/ psico.22.42.3508

Urrútia, G. y Bonfill, X. (2010). Declaración PRISMA: una propuesta para mejorar la publicación de revisiones sistemáticas y metaanálisis. Medicina Clínica, 135(11), 507511. https://doi.org/10.1016/j.medcli.2010.01.015

Roxana Angela Flores Flores es docente de la Institución Educativa IE0005 INDEPENDENCIA de Educación Inicial. Inició sus estudios el Instituto Superior Pedagógico Privado "Diego Thomson"; continuo estudios complementarios en la Universidad Nacional Mayor de San Marcos-UNMSM (Perú) en la Facultad de Educación. Para obtener el grado de Bachiller en 2010 y el Título de Licenciado en 2016. Se trasladó a la Universidad César Vallejo-UCV, para continuar sus estudios de posgrado, obteniendo el Grado de Magister en Educación. ORCID: https://orcid.org/0000-0003-4346-123X

Yolanda Josefina Huayta Franco es docente investigadora de la Universidad César Vallejo-UCV (Perú), en la Unidad de Posgrado de los programas de Maestrías y Doctorados. Inicio sus estudios superiores en la Universidad Inca Garcilaso de la Vega-UIGV (Perú), en la Facultad de Educación, en la especialidad de Ciencias Históricos Sociales y Filosóficas. Luego de lograr la licenciatura, se trasladó a la Universidad César Vallejo-UCV, para continuar sus estudios de posgrado, obteniendo el Grado de Magister en Educación con mención en la especialización en "Docencia y Gestión Educativa". Posee Grado de Doctora en Educación. Además, cuenta con Diplomados en "Formación de Especialistas en Entornos Virtuales de Aprendizaje" (UCV), “Tutoría en Entornos Virtuales de Aprendizaje" (UCV), "Estadística Aplicada a la Investigación Científica" en la Universidad San Martín de PorresUSMP, "Didáctica de Investigación en Posgrado" (UCV) en el año 2018. Docente visitante en la Universidad Mariana-UM (Colombia), en la Unidad de Maestría en Pedagogía. Está afiliada a la Universidad César Vallejo-UCV hasta la actualidad, con amplia experiencia en cátedra sobre investigación y dictado de experiencias curriculares de especialidad en ciencias de la educación, en las unidades de pregrado y posgrado, en programas tanto en educación, como en gestión pública. ORCID: https://orcid.org/0000-0003-0194-8891 
Ana Isabel Galindo Quispe es docente de la IE 7069 Cesar Vallejo (Perú), en la Especialidad de Ciencias Hisórico Sociales. Inicio sus estudios superiores en el Instituto Pedagógico Nacional de Monterrico, luego de lograr el Título de Profesora en la Especialidad de Ciencias Histórico Sociales, continuo estudios complementarios en la Universidad Nacional Mayor de San Marcos-UNMSM (Perú) en la Facultad de Educación para obtener el grado de Bachiller y el Título de Licenciado, se trasladó a la Universidad César Vallejo-UCV, para continuar sus estudios de posgrado, obteniendo el Grado de Magister en Educación con mención en "Administracion de la Educación". Además, cuenta con Segunda Especialidad en Formación Ciudadana y Cívica en la Universidad Peruana Unión-UPU y también en Gestión Escolar con Liderazgo Pedagógico en la Universidad Cayetano Heredia-UPCH y Diplomados en "Gestión escolar" en la UPCH. ORCID: https://orcid.org/0000-0003-01948891

Carolina del Pilar López Ruiz trabaja en el IESTP Manuel Seoane Corrales (Perú) como docente en los módulos de Actividades, Investigación e Idioma Extranjero. Inició sus estudios superiores en la Universidad Federico Villarreal-UFV (Perú), en la Facultad de Educación, en la especialidad de Cultura Física, luego de lograr la licenciatura, se trasladó a la Universidad César Vallejo-UCV, para continuar sus estudios de posgrado, obteniendo el Grado de Maestra en Educación con mención en la especialización en "Docencia y Gestión Educativa". Actualmente se encuentra terminando sus estudios de Doctorado en Educación en la UCV. Además, cuenta con estudios de nivel avanzado de inglés en el Instituto Cultural Peruano Norteamericano-ICPNA y docente del nivel avanzado de Danzas Folklóricas Nacionales en el Centro Folklórico del Magisterio "Alejandro Vivanco Guerra”. ORCID: https://orcid.org/0000-0002-9472-7651

Janeth Rosario Gutiérrez Rojas es docente de la IE 6070 Héroes del Alto Cenepa (Perú), en la Especialidad de Matemática y Física. Inicio sus estudios superiores en la Universidad Nacional Mayor de San Marcos-UNMSM, luego de lograr el Bachiller y título de Profesora en la Especialidad de Matemática y Física para continuar sus estudios de posgrado, obteniendo el Grado de Magister en Educación con mención en "Administracion de la Educación”. ORCID: https://orcid.org/0000-0001-9253-9588 